\title{
Two-Step Mechanism for Low-Temperature Oxidation of Vacancies in Graphene
}

\author{
Johan M. Carlsson, ${ }^{1, *}$ Felix Hanke, ${ }^{1}$ Suljo Linic,,${ }^{1,2}$ and Matthias Scheffler ${ }^{1}$ \\ ${ }^{1}$ Fritz-Haber-Institut der Max-Planck-Gesellschaft, Faradayweg 4-6, D-14195 Berlin, Germany \\ ${ }^{2}$ Department of Chemical Engineering, University of Michigan, Ann Arbor, Michigan 48109-2136, USA \\ (Received 25 July 2008; revised manuscript received 22 February 2009; published 23 April 2009)
}

\begin{abstract}
We study the oxidation of vacancies in graphene by ab initio atomistic thermodynamics to identify the dominant reaction mechanisms. Our calculations show that the low-temperature oxidation occurs via a two-step process: Vacancies are initially saturated by stable $\mathrm{O}$ groups, such as ether (C-O-C) and carbonyl $(\mathrm{C}=\mathrm{O})$. The etching is activated by a second step of additional $\mathrm{O}_{2}$ adsorption at the ether groups, forming larger $\mathrm{O}$ groups, such as lactone $(\mathrm{C}-\mathrm{O}-\mathrm{C}=\mathrm{O})$ and anhydride $(\mathrm{O}=\mathrm{C}-\mathrm{O}-\mathrm{C}=\mathrm{O})$, that may desorb as $\mathrm{CO}_{2}$ just above room temperature. Our studies show that the partial pressure of oxygen is an important external parameter that affects the mechanisms of oxidation and that allows us to control the extent of etching.
\end{abstract}

Oxidation of carbon is one of the most important chemical reactions. Complete combustion of solid carbon materials to form $\mathrm{CO}_{2}$ is important for heating, regeneration of catalysts poisoned by carbon, and oxidation of diesel soot. On the other hand, partial oxidation of carbon is critical for the controlled formation of nanopatterns on the surface of graphite [1]. These nanopatterns may be used as casting frames for nanoparticles [2]. Controlled oxidation of carbon materials is also used to add functional oxygen (O) groups to the surface and tune its chemical activity [3]. While controlling the process of carbon oxidation is crucial, the process has been a subject of considerable controversy as various experimental studies have highlighted different aspects of the reaction and thereby yielded contradictory results.

Scanning tunneling microscopy (STM) experiments, for instance, demonstrate that the defect-free regions of the basal plane of graphite are inert towards $\mathrm{O}_{2}$ below a critical temperature of $875 \mathrm{~K}$ [4]. It has also been shown that carbon vacancies grow into larger openings on the basal plane of graphite under atmospheric air conditions [4,5]. However, the details of the evolution of the graphite surface structure during the process of oxidation is unclear as STM is unable to distinguish between carbon and oxygen atoms. Temperature programmed desorption (TPD) measurements, performed by first oxidizing a graphite sample in atmospheric air and subsequently heating under ultrahigh vacuum (UHV) conditions, have confirmed the activation of molecular oxygen and attachment of oxygen atoms on defective graphite. However, the main product in these TPD spectra was CO, contrary to the release of $\mathrm{CO}_{2}$ during oxidation under atmospheric air [6].

The carbon oxidation reaction has also been investigated in a number of theoretical contributions. These investigations have concentrated on graphene, as the interaction between the layers in graphite is so small that considering only a single graphene sheet gives only negligible differences. A few of the theoretical works have addressed the etching of vacancies $[7,8]$, while the majority have con- centrated on the oxidation of graphite edges [9-13]. These studies have led to a mechanism for incomplete combustion, based on $\mathrm{O}_{2}$ dissociation at bare edges and direct $\mathrm{CO}$ desorption [9]. This mechanism is somewhat contradictory to the experimental observations of complete combustion leading to $\mathrm{CO}_{2}$ under atmospheric oxygen pressure. These studies also do not explain the results of isotopic exchange studies, where different isotopes of oxygen were reacted with graphite, which showed that the formation of different oxygen structures on graphite was a consequence of a number of sequential reactions [6]. Summarizing previous measurements indicates that the process of the oxidation of graphitic carbon is pressure-dependent and that various mechanisms may be operating under different conditions.

We carried out a systematic study of oxidation of vacancies in graphene using ab initio atomistic thermodynamics (AIATD) [14]. Graphene was chosen as the model system, but the results are also applicable to other $s p^{2}$-bonded carbon materials such as graphite and nanotubes. The aim is to provide a coherent mechanism for lowtemperature oxidation in dry air without water or hydrogen, which can reconcile the $\mathrm{CO}_{2}$ formation during complete combustion with the observation of dominant $\mathrm{CO}$ desorption in TPD experiments after oxidation. The reaction is divided into finding the thermodynamically most favorable $\mathrm{O}$ groups at vacancies in a constrained equilibrium and calculating the binding energy for desorption of $\mathrm{CO}$ and $\mathrm{CO}_{2}$ to determine the relative stability of these $\mathrm{O}$ groups. Combining these elementary steps finally leads to a two-step mechanism for the oxidative etching of vacancies in graphene.

In the AIATD method, the free energy of formation $\Delta G_{\text {form }}$ of a given system in equilibrium with a gas phase reservoir is calculated as a function of $T$ and $p$ :

$$
\begin{aligned}
\Delta G_{\text {form }}\left(n_{\mathrm{C}}, n_{\mathrm{O}}, T, p\right)= & E^{\text {motif }}\left(n_{\mathrm{C}}, n_{\mathrm{O}}\right) \\
& -n_{\mathrm{O}} \mu_{\mathrm{O}}-\left(E^{G}-n_{\mathrm{C}} \mu_{\mathrm{C}}\right) \\
& +\Delta F^{\mathrm{vib}}(T)-k_{B} T \ln g^{\text {config }},
\end{aligned}
$$


where $E^{G}$ and $E^{\text {motif }}$ refer to the total energy of a graphene sheet before and after the oxidized vacancy are created, respectively, $n_{\mathrm{C}}$ denotes the number of $\mathrm{C}$ atoms that have been exchanged with the bulk reservoir $\mu_{\mathrm{C}}$, and $n_{\mathrm{O}}$ corresponds to the number of $\mathrm{O}$ atoms having the chemical potential $\mu_{\mathrm{O}}$ determined by the gas phase molecules. $\Delta F^{\mathrm{vib}}(T)$ is the additional vibrational free energy of the vacancies. Since it is mainly due to the adsorption of oxygen, we include only the bending and stretch modes of the $\mathrm{O}$ groups attached to the vacancy. The calculated stretch frequencies for these $\mathrm{O}$ groups all fall within the frequency regions assigned to the corresponding group by IR measurements [15]. Finally, $g^{\text {config }}$ is the configurational degeneracy of the vacancies, obtained by exact enumeration of all possible configurations.

The total energy calculations are based on densityfunctional theory (DFT) in the generalized gradient approximation [16], as implemented in the CASTEP code [17]. Key results were also confirmed in the local density approximation. We have optimized ultrasoft pseudopotentials for a plane-wave energy cutoff of $550 \mathrm{eV}$. The oxidized vacancies were modeled in a $6 \times 6$ supercell (containing 72 atoms in pure graphene), with a layer separation of $15 \AA$ and a Brillouin zone sampling of $(2 \times 2 \times 1)$. All atomic positions were relaxed until the maximal force on each atom was smaller than $0.01 \mathrm{eV} / \AA$. We have performed reference all-electron calculations for selected configurations with the FHI-AIMS code [18] to test the pseudopotential approximation and find identical geometries and formation energy differences of no more than $0.01 \mathrm{eV}$. Our estimate of the numerical inaccuracy of the calculated formation energies is $\approx 0.1 \mathrm{eV}$.

We start our analysis by studying oxygen adsorption on the basal plane of the graphene sheet. Our calculations demonstrate that the $\mathrm{O}_{2}$ dissociation on the defect-free regions would proceed through the metastable adsorbate state (on the top site) in a process called cyclo addition. The most favorable adsorption site for individual $\mathrm{O}$ atoms on the graphene sheet is the bridge site (forming an epoxy group) in agreement with previous DFT calculations $[19,20]$. Nevertheless, the $\mathrm{O}_{2}$ dissociation is strongly endothermic (see route $G$ in Fig. 1), consistent with the observation that the basal plane is inert towards the activation of $\mathrm{O}_{2}$ below the critical temperature $[4,5]$.

On the contrary, vacancies in graphene are very reactive towards $\mathrm{O}_{2}$. We have previously studied such vacancies demonstrating that the lowest energy structures are organized so that the number of carbon dangling bonds is minimized by the formation of pentagons surrounding the vacancies [21]. Figure 1 shows that the pentagons at a four-atom carbon vacancy (V4) interact very strongly with $\mathrm{O}_{2}$, such that they are saturated by ether (-C-O-C-) groups in a highly exothermic process, following route I. These results are consistent with recent DFT calculations that showed that the $\mathrm{O}_{2}$ dissociation has a negligible barrier

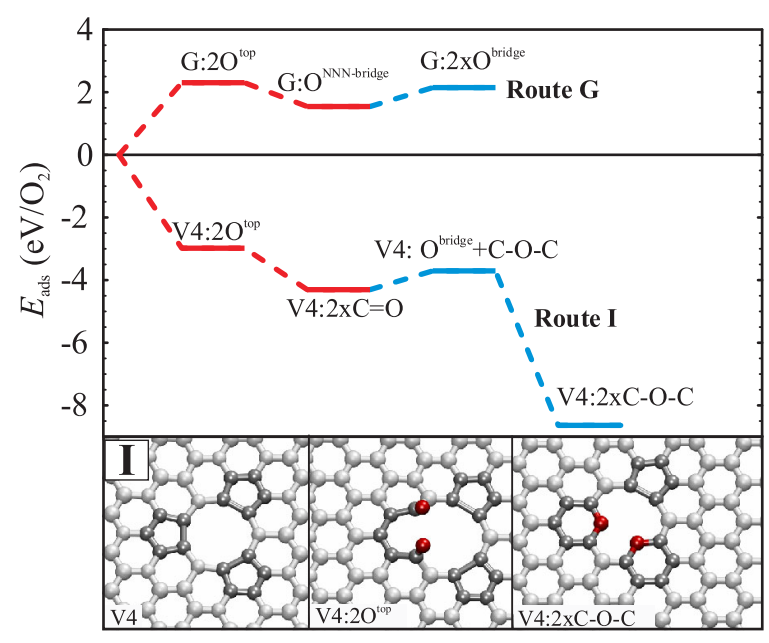

FIG. 1 (color online). Upper panel: The adsorption energy for dissociative $\mathrm{O}_{2}$ adsorption on the perfect basal plane of graphene (route $\mathrm{G}$ ) and at a bare four-atom (V4) vacancy (route I). Red lines indicate $\mathrm{O}_{2}$ adsorption and blue lines $\mathrm{O}$-atom diffusion. Lower panel: The corresponding geometries of the stable intermediate adsorption structures along route I, summarizing the first step of the oxidation mechanism. Oxygen adsorption at a pentagon leads to formation of two parallel carbonyl $(\mathrm{C}=\mathrm{O})$ groups. The adsorption energy for $\mathrm{O}$ atoms forming ether groups is larger than for carbonyl groups, such that $\mathrm{O}$ atoms are expected to diffuse along the perimeter of the vacancy to form another ether group. All molecular images were done with VMD [22].

at a V1 vacancy [7] and that the final state in the $\mathrm{O}_{2}$ dissociation at a V2 vacancy results in the formation of ether groups [8]. A decomposition of $\Delta G_{\text {form }}$ furthermore shows that the interaction between the neighboring ether groups is negligible, such that the attachment of $\mathrm{O}$ atoms can proceed until complete saturation. In general, we find that at even vacancies (vacancies with an even number of removed $\mathrm{C}$ atoms) all pentagons at the vacancies are saturated by ether groups. At odd vacancies, mainly ether groups are formed with a remaining undercoordinated $\mathrm{C}$ atom forming a carbonyl $(-\mathrm{C}=\mathrm{O})$ group. We identify this saturation of vacancies by formation of ether or carbonyl groups as the first step in the oxidation process.

The oxygen-saturated vacancies may appear passivated, but Fig. 2 reveals that the ether groups provide additional $\mathrm{O}_{2}$ dissociation sites. These sites are less reactive than bare vacancies but considerably more reactive than the graphene basal plane. Figure 2 shows that the adsorption energies for the additional oxygen atoms on the graphene structures, which are rich in ether groups, are highly exothermic. This forms $\mathrm{O}$ groups such as the lactone $(-\mathrm{C}-\mathrm{O}-\mathrm{C}=\mathrm{O})$ and the anhydride $(\mathrm{O}=\mathrm{C}-\mathrm{O}-\mathrm{C}=\mathrm{O})$ group. We identify this process as a second step in the oxidation mechanism. Note that single $\mathrm{O}$ atoms diffusing around the vacancy may also attach to the lactone group to form a carbonate $[\mathrm{O}-(\mathrm{C}=\mathrm{O})-\mathrm{O}]$, not shown. 


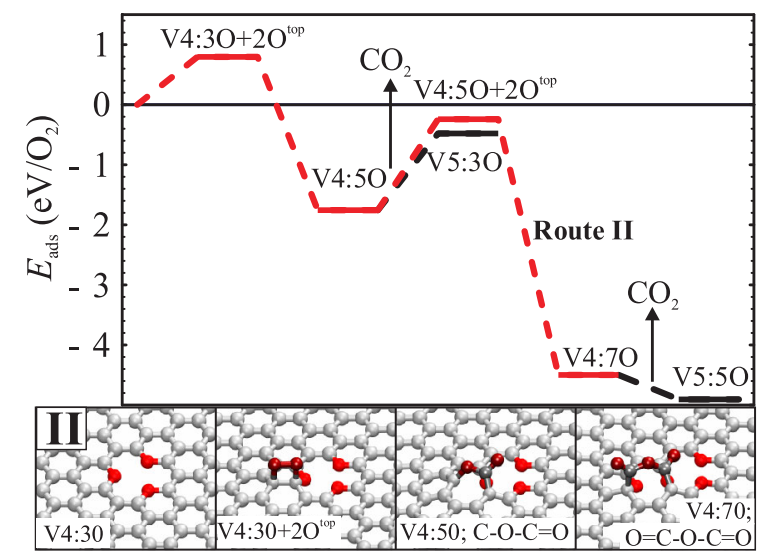

FIG. 2 (color online). Upper panel: The adsorption energy for dissociative $\mathrm{O}_{2}$ adsorption at an oxygen-saturated V4:3O vacancy along route II. Red lines indicate $\mathrm{O}_{2}$ adsorption and black lines $\mathrm{CO}_{2}$ desorption. Lower panel: The corresponding geometries of the stable intermediate adsorption structures. The first event is the formation of a lactone $(\mathrm{C}-\mathrm{O}-\mathrm{C}=\mathrm{O})$ group, which can either desorb as $\mathrm{CO}_{2}$ or form an anhydride $(\mathrm{O}=\mathrm{C}-\mathrm{O}-\mathrm{C}=\mathrm{O})$ group by additional adsorption of an $\mathrm{O}_{2}$. The latter can finally disintegrate by releasing $\mathrm{CO}_{2}$.

The equilibrium concentration of the different $\mathrm{O}$ groups is determined by the structure that has the lowest $\Delta G_{\text {form }}$. We consider a constrained equilibrium, where only the $\mathrm{O}_{2}$ adsorption/desorption can take place. No variation in vacancy size was allowed as $\mathrm{CO}$ or $\mathrm{CO}_{2}$ desorption is not considered at this stage. The chemical potential of oxygen is considered as a variable determined by the external conditions, while the chemical potential of the carbon atoms is determined by the graphene layer.

An inspection of the formation energy diagram in Fig. 3 and the structures associated with various curves on this diagram shows that, for all examined vacancy sizes (V1-V6), the two-step oxidation mechanism presented in Figs. 1 and 2 plays the dominant role. At very low oxygen chemical potential (characterized by low oxygen pressure and high temperature), vacancies are saturated with ether groups (see route $G$ in Fig. 1). As the oxygen chemical potential is increased, additional $\mathrm{O}$ atoms are attached to the vacancy (see route II in Fig. 2). $\Delta G_{\text {form }}$ may even become negative at larger values of $\mu_{\mathrm{O}}$, which suggests a spontaneous vacancy formation.

We can also relate $\mu_{\mathrm{O}}$ to a temperature and pressure scale to obtain the phase diagram for a particular vacancy. Such a phase diagram for a V4 vacancy is shown in Fig. 4. This phase diagram indicates that the V4 vacancy would remain without any $\mathrm{O}$ groups only at extremely low $\mathrm{O}$ pressures and high temperatures. A higher $\mathrm{O}$ pressure and/or lower temperature leads to a progressive saturation of the vacancy until the V4:30 configuration, characterized by three pentagons filled by one ether group each, is formed. Correlating the formation energy of the oxygen functional groups with an analysis of Fig. 4 reveals that

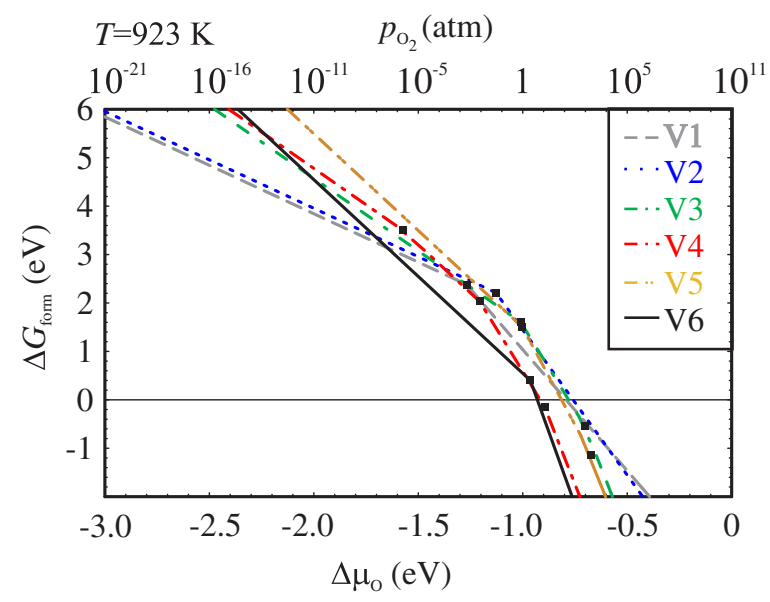

FIG. 3 (color online). The minimal formation energy $\Delta G_{\text {form }}$ for the oxidized multiple vacancies V1-V6 as a function of the oxygen chemical potential $\Delta \mu_{\mathrm{O}}=\mu_{\mathrm{O}}-\mu_{\mathrm{O}}^{\mathrm{DFT}}$ at a typical oxidation temperature $T=923 \mathrm{~K}[1,4,5] . \mu_{\mathrm{O}}^{\mathrm{DFT}}$ is the chemical potential of an $\mathrm{O}$ atom in the free molecule calculated by DFT. The slope of the lines is given by the number of $\mathrm{O}$ atoms adsorbed at the vacancy, and the black squares indicate the transition value of the $\mu_{\mathrm{O}}$, where the configuration changes. The upper scale converts $\mu_{\mathrm{O}}$ into a pressure scale.

increasing the oxygen concentration beyond the $\mathrm{V} 4: 3 \mathrm{O}$ results in the formation of oxygen complexes containing anhydride and ether groups.

The number of $\mathrm{O}$ groups present at vacancies also depends on their stability with respect to decomposition via $\mathrm{CO}$ and $\mathrm{CO}_{2}$ desorption. We calculated the binding energy for $\mathrm{CO}$ and $\mathrm{CO}_{2}$ desorption from different $\mathrm{O}$ groups shown in Fig. 5. The values were converted into a critical temperature $T_{c}$ defined by $\Delta G_{\text {bind }}\left(T_{c}, p\right)=0$, above which less than half of the $\mathrm{O}$ groups would remain intact. This analysis shows that the $\mathrm{O}$ groups form a stability hierarchy.

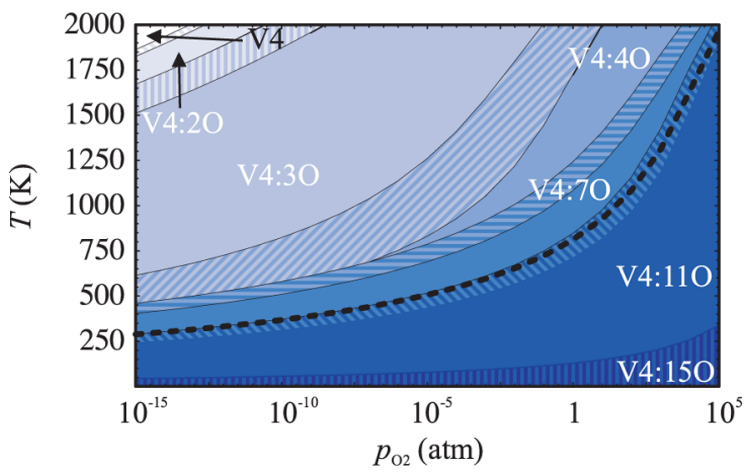

FIG. 4 (color online). A phase diagram for a V4 vacancy in graphene, showing how the oxygen concentration changes as a function of $T$ and $p$. V4:nO denotes how many $\mathrm{O}$ atoms are adsorbed at the vacancy. $\Delta G_{\text {form }}$ is negative below the dotted black line. The hashed regions indicate coexistence regions, where the energy difference between two configurations is below the numerical resolution. 


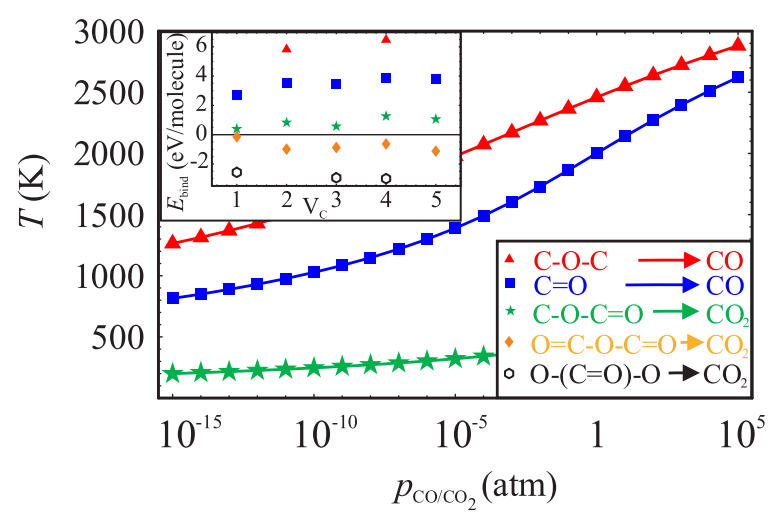

FIG. 5 (color online). The binding energy and stability range of the $\mathrm{O}$ groups with respect to $\mathrm{CO}$ or $\mathrm{CO}_{2}$ desorption. $\Delta G_{\text {bind }}(T, p)$ is negative below the critical temperature $T_{c}$, indicated by the lines. The inset shows the binding energy $E_{\text {bind }}$ for $\mathrm{CO}$ or $\mathrm{CO}_{2}$ desorption at multiple vacancies V1-V5 in graphene.

The ether group has a large binding energy, such that it is likely to be present in the material even after high temperature annealing and they may not be visible in TPD measurements. The carbonyl group has a smaller binding energy, such that it can be detected as a CO-signal in the high temperature region of a TPD spectrum. The lactone group $(-\mathrm{C}-\mathrm{O}-\mathrm{C}=\mathrm{O})$ is more volatile, and its concentration at the vacancies decreases even just slightly above room temperature. The large binding energies for these groups are due to the unfavorable empty pentagons left behind after desorption. The local geometry of the $\mathrm{O}$ groups at the vacancies differs from those at the edges of graphitic basal planes. For example, anhydride and carbonate groups are bent out of the basal plane of graphene, which reduces the binding energy for these groups at the vacancies (in contrast to the endothermic values reported at edges [13]), suggesting that these large $\mathrm{O}$ groups desorb from vacancies soon after formation.

In summary, our analysis suggests that low-temperature etching of vacancies in graphene proceeds through a twostep mechanism. Bare vacancies are quickly saturated by ether and carbonyl groups following route I in Fig. 1. These $\mathrm{O}$ groups are stable with respect to $\mathrm{CO}$-desorption below the critical temperature $T_{c}$, and they can be considered as the ground state in an oxygen atmosphere. The discrepancy between observed dominant desorption of $\mathrm{CO}_{2}$ during the etching of vacancies at atmospheric oxygen pressure and the dominant CO-desorption pathways observed in TPD experiments can be explained by the pressure gap. TPD measurements probe the ether and carbonyl groups which are stable at UHV conditions. The etching of the vacancies, on the contrary, proceeds at atmospheric $\mathrm{O}$ pressures, such that the $\mathrm{O}_{2}$ dissociation at the ether groups can be acti- vated. This leads to the formation of larger $\mathrm{O}$ groups, primarily lactones, that desorb directly as $\mathrm{CO}_{2}$ or that are further activated by additional $\mathrm{O}_{2}$ dissociation forming anhydride groups. These groups decompose rapidly, and the $\mathrm{CO}_{2}$ desorption exposes new sites for $\mathrm{O}_{2}$ dissociation, thus driving the etching reaction further.

*johanc@fhi-berlin.mpg.de

[1] A. Böttcher, M. Heil, N. Stürzl, S. S. Jester, S. Malik, F. Perez-Willard, P. Brenner, D. Gerthsen, and M. Kappes, Nanotechnology 17, 5889 (2006).

[2] H. Hoevel, T. Becker, A. Bettac, B. Reihl, M. Tchudy, and E. J. Williams, J. Appl. Phys. 81, 154 (1997).

[3] G. Mestl, N. I. Maksimova, N. Keller, V. V. Roddatis, and R. Schlögl, Angew. Chem., Int. Ed. 40, 2066 (2001).

[4] J. R. Hahn, Carbon 43, 1506 (2005).

[5] F. Stevens, L. A. Kolodny, and T. P. Beebe, Jr., J. Phys. Chem. B 102, 10799 (1998).

[6] B. Marchon, J. Carazza, H. Heinemann, and G. Samorjai, Carbon 26, 507 (1988).

[7] A. Allouche and Y. Ferro, Carbon 44, 3320 (2006).

[8] S. M. Lee, Y.H. Lee, Y. G. Hwang, J. R. Hahn, and H. Kang, Phys. Rev. Lett. 82, 217 (1999).

[9] N. Chen and R. T. Yang, J. Phys. Chem. A 102, 6348 (1998).

[10] A. Incze, A. Pasturel, and C. Chatillon, Surf. Sci. 537, 55 (2003).

[11] K. Sendt and B.S. Heynes, J. Phys. Chem. C 111, 5465 (2007).

[12] O. Hod, V. Barone, J. E. Peralta, and G. E. Scuseria, Nano Lett. 7, 2295 (2007).

[13] A. Sanchez and F. Mondragon, J. Phys. Chem. C 111, 612 (2007).

[14] E. Kaxiras, Y. Bar-Yam, J. D. Joannopoulos, and K. C. Pandey, Phys. Rev. B 35, 9625 (1987); M. Scheffler and J. Dabrowski, Philos. Mag. A 58, 107 (1988); K. Reuter and M. Scheffler, Phys. Rev. B 65, 035406 (2001).

[15] P.E. Fanning and M. A. Vannice, Carbon 31, 721 (1993).

[16] J. P. Perdew, K. Burke, and M. Ernzerhof, Phys. Rev. Lett. 77, 3865 (1996).

[17] The CASTEP code: S. J. Clark, M. D. Segall, C. J. Pickard, P. J. Hasnip, M. J. Probert, K. Refson, and M. Payne, Z. Kristallogr. 220, 567 (2005).

[18] The AIMS code: V. Blum, R. Gehrke, F. Hanke, P. Havu, V. Havu, X. Ren, K. Reuter, and M. Scheffler, Comput. Phys. Commun. (to be published).

[19] S.-P. Chan, G. Chen, X. G. Gong, and Z.-F. Liu, Phys. Rev. Lett. 90, 086403 (2003).

[20] D. Lamoen and B. N. J. Persson, J. Chem. Phys. 108, 3332 (1998).

[21] J. M. Carlsson and M. Scheffler, Phys. Rev. Lett. 96, 046806 (2006).

[22] The VMD program: W. Humphrey, A. Dalke, and K. Schulten, J. Mol. Graphics 14, 33 (1996). 